\title{
MHD Effect on an Oscillatory Mixed Convective Flow Past an Infinite Vertical Porous Plate with Radiation
}

\author{
Nazibuddin Ahmed, Sujan Sinha \\ Department of Mathematics, Gauhati University, Guwahati, India \\ Email: saheel_nazib@yahoo.com,mathssujangu@gmail.com \\ Received August 17, 2013; Revised September 17, 2013; accepted September 25, 2013
}

Copyright (C) 2013 Nazibuddin Ahmed, Sujan Sinha. This is an open access article distributed under the Creative Commons Attribution License, which permits unrestricted use, distribution, and reproduction in any medium, provided the original work is properly cited.

\begin{abstract}
A parametric study to investigate the effects of magnetic field and radiation on a mixed convective flow past an infinite vertical porous plate with constant suction velocity when the free stream varies periodically with time about a steady mean is presented. A uniform magnetic field is applied normal to the plate. The Rosseland approximation is used to describe the radiative heat flux in the energy equation and the resultant set of non-dimensional equations are solved analytically by adopting regular perturbation technique. The profiles of velocity, temperature, skin-friction and nusselt number are demonstrated graphically for various values of the parameters involved in the problem and the results are physically interpreted.
\end{abstract}

Keywords: Mixed Convection; Porous Medium; Radiation

\section{Introduction}

The problems of interaction of free and forced convection with thermal radiation of viscous incompressible MHD unsteady flow past infinite vertical plates with mass transfer are being studied nowadays due to many applications of such problems in Astrophysics, Geophysical and different Engineering fields. The heating of rooms and buildings by use of radiators is an example of heat transfer by free convection. The study of MHD is quite important in the field of aeronautics, especially in missile aerodynamics, since the temperature that occurs in such flight speeds are sufficient to dissociate or even ionize the air appreciably. For example, when a high speed missile re-enters the earth's atmosphere, a very large amount of heat is generated due to the friction of air molecules and this viscous heating may some times be so considerable as to ionize the air near the stagnation point. This ionized gas or plasma interacts with the magnetic field and alter the heat transfer and friction characteristics. In addition to this, some fluids can also emit and absorb thermal radiation. In such a situation, the study of the effect of the magnetic field on the flow characteristics becomes more interesting as the fluid is not only electrical conductor but also capable of emitting and absorbing thermal radiation. This type of investigation is carried out because of its importance in space and temperature re- lated problems.

MHD is the science of motion of electrically conducting fluid in presence of magnetic field. There are numerous examples of application of MHD principles, including MHD generators, MHD pumps and MHD flow meters etc. The dynamo and motor is a classical example of MHD principle. MHD principles also find its application in medicine and biology. The present form of MHD is due to the pioneer contribution of several authors like Alfven [1], Cowling [2], Crammer and Pai [3], Shercliff [4] and Ferraro and Pulmption [5]. Model studies on MHD free and forced convection with heat and mass transfer problems have been carried out by many of the authors due to their application in many branches of science and technology. Some of them are Ahmed [6], Elbashbeshy [7] and Singh and Singh [8]. Gregantopoulos et al. [9] studied two-dimensional unsteady free convection and mass transfer flow of an incompressible viscous dissipative and electrically conducting fluid past an infinite vertical porous plate.

Radiation is a process of heat transfer through electromagnetic waves. Radiative convective flows are encountered in countless industrial and environment process. For e.g. heating and cooling chambers, fossil fuel combustion energy processes, evaporation from large open water reservoirs, astrophysical flows etc. Radiative 
heat and mass transfer play an important role in manufacturing industries for the design of reliable equiponent. If the temperature of the surrounding field is rather high, radiation effect plays an important role in space related technology. The effect of radiation on various convective flows under different conditions has been studied by many researchers including Hussain and Takhar [10], Ahmed and Sarmah [11], Rajesh and Varma [12] and Ahmed et al. [13]. As the present authors are aware till now no attempt has been made to study the magnetic field effect on an oscillatory mixed convective flow past an infinite vertical porous plate with radiation following the technique adopted by Mansour [14]. Such an attempt has been made in the present paper.

The main objective of the present investigation is to study the MHD effects on the unsteady mixed convective mass transfer flow past an infinite vertical porous plate with constant suction, where the plate temperature oscillates with the same frequency as that of variable suction velocity. It is assumed that the plate is embedded in a uniform porous medium and moves with a constant velocity in the direction of the flow in presence of a transverse magnetic field. This work is an extension to the work done by Mansour [14] to consider the effect of MHD.

\section{Mathematical Analysis}

We now consider an MHD conducting flow of a viscous incompressible electrically conducting fluid past an infinite vertical porous plate with constant suction under the influence of a uniform transverse magnetic field. Our investigation is restricted to the following assumptions:

- The polarization effects are assumed to be negligible and hence the electric field is also negligible.

- The suction velocity is constant.

- The variations of all fluid properties other than the variations of density except in so far as they give rise to a body force are ignored completely.

- All the physical variables are functions of $y^{\prime}$ and $t^{\prime}$ only as the plate are infinite.

- It is assumed that the variation of expansion co-efficient is negligibly small and the pressure and influence of the pressure on the density are negligible.

- The free stream oscillates about a steady mean.

We introduce a co-ordinate system $\left(x^{\prime}, y^{\prime}, z^{\prime}\right)$ with $X$ axis vertically upwards along the plate, $Y$-axis perpendicular to the plate and directed into the fluid region and $Z$-axis along the width of the plate as shown in Figure 1. Let the components of velocity along with $X$ and $Y$ axes should be $u^{\prime}$ and $v^{\prime}$. Let these velocity components are chosen in the upward direction along the plate and normal to the plate respectively.

Under these assumptions, the equations that describe the physical situation are given by

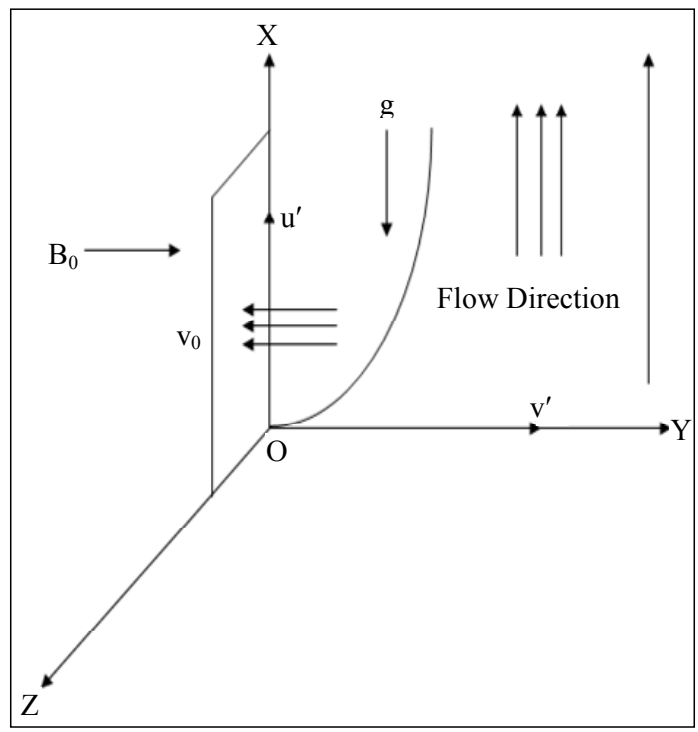

Figure 1. Physical model of the problem.

$$
\frac{\partial v^{\prime}}{\partial y^{\prime}}=0
$$

$$
\begin{aligned}
\frac{\partial u^{\prime}}{\partial t^{\prime}}+v^{\prime} \frac{\partial u^{\prime}}{\partial y^{\prime}}= & -\frac{1}{\rho^{\prime}} \frac{\partial p^{\prime}}{\partial x^{\prime}}+v \frac{\partial^{2} u^{\prime}}{\partial y^{\prime 2}}+g \beta\left(T^{\prime}-T_{\infty}^{\prime}\right) \\
& -v \frac{u^{\prime}}{K^{\prime}}-\frac{\sigma B_{0}^{2} u^{\prime}}{\rho^{\prime}}
\end{aligned}
$$

$$
\begin{aligned}
& \frac{\partial v^{\prime}}{\partial t^{\prime}}=-\frac{1}{\rho} \frac{\partial p^{\prime}}{\partial y^{\prime}} \\
& \frac{\partial T^{\prime}}{\partial t^{\prime}}+v^{\prime} \frac{\partial T^{\prime}}{\partial y^{\prime}}=\alpha\left(\frac{\partial^{2} T^{\prime}}{\partial y^{\prime 2}}-\frac{1}{K_{T}} \frac{\partial q_{r}}{\partial y^{\prime}}\right)
\end{aligned}
$$

All the physical quantities involved in the above equations are defined in the nomenclature.

The boundary conditions are:

$$
\begin{aligned}
& u^{\prime}=0, T^{\prime}=T_{w}^{\prime} \text { at } y^{\prime}=0 \\
& u^{\prime} \rightarrow U^{\prime}(t)=U_{0}\left(1+\varepsilon \mathrm{e}^{i \omega^{\prime} t^{\prime}}\right), T^{\prime} \rightarrow T_{\infty}^{\prime} \text { as } y^{\prime} \rightarrow \infty
\end{aligned}
$$

As there is a constant suction velocity $v_{0}$ at the plate, Equation (1) yields

$$
v^{\prime}=-v_{0}
$$

where $v_{0}$ is a scale of suction velocity which is nonzero positive constant. The negative sign indicates that the suction is towards plate.

Outside the boundary layer, Equation (2) gives

$$
-\frac{1}{\rho^{\prime}} \frac{\partial p^{\prime}}{\partial x^{\prime}}=\frac{\mathrm{d} U^{\prime}}{\mathrm{d} t^{\prime}}+\frac{v}{K^{\prime}} U^{\prime}+\frac{\sigma B_{0}^{2} U^{\prime}}{\rho^{\prime}}
$$

In order to write the governing equations and the boundary conditions in dimensional form, the following non-dimensional quantities are introduced: 


$$
\begin{aligned}
& u=\frac{u^{\prime}}{U_{0}}, y=\frac{y^{\prime} v_{0}}{v}, t=\frac{t^{\prime} v_{0}^{2}}{4 v}, G r=\frac{v g \beta\left(T_{w}^{\prime}-T_{\infty}^{\prime}\right)}{U_{0} v_{0}^{2}}, \\
& \theta=\frac{T^{\prime}-T_{\infty}^{\prime}}{T_{w}^{\prime}-T_{\infty}^{\prime}}, \operatorname{Pr}=\frac{v}{\alpha}, M=\frac{\sigma B_{0}{ }^{2} v}{\rho v_{0}{ }^{2}}, \\
& \omega=\frac{4 v \omega^{\prime}}{v_{0}^{2}}, U=\frac{U^{\prime}}{U_{0}}, C_{T}=\frac{T_{w}^{\prime}}{T_{w}^{\prime}-T_{\infty}^{\prime}}, \\
& R=\frac{4 \sigma^{\prime}\left(T_{w}^{\prime}-T_{\infty}^{\prime}\right)^{3}}{k K_{T}}, K=\frac{K^{\prime} v_{0}^{2}}{v^{2}}
\end{aligned}
$$

In view of the Equations (6)-(8), the Equations (2)-(4) reduce to the following dimensional form:

$$
\begin{aligned}
& \frac{1}{4} \frac{\partial u}{\partial t}-\frac{\partial u}{\partial y}=\frac{1}{4} \frac{\mathrm{d} U}{\mathrm{~d} t}+\frac{\partial^{2} u}{\partial y^{2}}+\operatorname{Gr} \theta+N(U-u) \\
& \frac{1}{4} \frac{\partial \theta}{\partial t}-\frac{\partial \theta}{\partial y} \\
& =\left[1+\frac{4}{3} R\left(\theta+C_{T}\right)^{3}\right] \frac{\partial^{2} \theta}{\partial y^{2}}+4 R\left(\theta+C_{T}\right)^{2}\left(\frac{\partial \theta}{\partial y}\right)^{2}
\end{aligned}
$$

The corresponding boundary conditions are:

$$
\begin{aligned}
& u=0, \theta=1 \text { at } y=0 \\
& u=U(t), \theta=0 \text { as } y \rightarrow \infty
\end{aligned}
$$

\section{Method of Solution}

Equations (9) and (10) are coupled non-linear partial differential and these cannot be solved in closed-form. However, these equations can be reduced to a set of ordinary differential equations, which can be solved analytically. This can be done by representing the velocity, temperature and concentration of the fluid in the neighbourhood of the plate as

$$
\begin{aligned}
& u=u_{0}(y)+\varepsilon \mathrm{e}^{i \omega t} u_{1}(y)+o\left(\varepsilon^{2}\right)+\cdots \\
& \theta=\theta_{0}(y)+\varepsilon \mathrm{e}^{i \omega t} \theta_{1}(y)+o\left(\varepsilon^{2}\right)+\cdots \\
& U=1+\varepsilon \mathrm{e}^{i \omega t}
\end{aligned}
$$

Substituting (12) in Equations (9) and (10), equating the harmonic terms and neglecting the higher order terms of $0\left(\varepsilon^{2}\right)$, we obtain

$$
\begin{aligned}
& u_{0}^{\prime \prime}+u_{0}^{\prime}-N u_{0}=-N-G r \theta_{0} \\
& u_{1}^{\prime \prime}+u_{1}^{\prime}-\left(N+\frac{i \omega}{4}\right) u_{1}=-N-G r \theta_{1}-\frac{i \omega}{4} \\
& {\left[1+\frac{4}{3} R\left(\theta_{0}+C_{T}\right)^{3}\right] \theta_{0}^{\prime \prime}+4 R\left(\theta_{0}+C_{T}\right)^{2} \theta_{0}^{\prime}} \\
& +\operatorname{Pr} \theta_{0}=0
\end{aligned}
$$

$$
\begin{aligned}
& {\left[1+\frac{4}{3} R\left(\theta_{0}+C_{T}\right)^{3}\right] \theta_{1}^{\prime \prime}+8 R\left(\theta_{0}+C_{T}\right)^{2}} \\
& \left(\frac{\partial \theta_{0}}{\partial y}\right)\left(\frac{\partial \theta_{1}}{\partial y}\right)+8 R\left(\theta_{0}+C_{T}\right)^{2}\left(\frac{\partial \theta_{0}}{\partial y}\right)^{2} \theta_{1} \\
& +4 R\left(\theta_{0}+C_{T}\right)^{2} \theta_{0}^{\prime \prime} \theta_{1}+\operatorname{Pr} \theta_{1}^{\prime}-\frac{i \omega}{4} \operatorname{Pr} \theta_{1}=0
\end{aligned}
$$

where primes denotes ordinary differentiation with respect to $y$.

The corresponding boundary conditions can be written as

$$
\begin{aligned}
& u_{0}=0, u_{1}=0, \theta_{0}=1, \theta_{1}=0 \text { at } y=0 \\
& u_{0} \rightarrow 1, u_{1} \rightarrow 1, \theta_{0} \rightarrow 0, \theta_{1} \rightarrow 0 \text { as } y \rightarrow \infty
\end{aligned}
$$

If we assume the radiation parameter $R$ to be small, we expand the velocity and temperature as

$$
\begin{aligned}
& u_{0}(y)=u_{01}(y)+R u_{02}(y), u_{1}(y)=u_{11}(y)+R u_{12}(y) \\
& \theta_{0}(y)=\theta_{01}(y)+R \theta_{02}(y), \theta_{1}(y)=\theta_{11}(y)+R \theta_{12}(y)
\end{aligned}
$$

Substituting (18) in Equations (13)-(16), we obtain the following set of equations for the mean flow:

$$
\begin{aligned}
& u_{01}^{\prime \prime}+u_{01}^{\prime}-N u_{01}=-N-G r \theta_{01} \\
& u_{02}^{\prime \prime}+u_{02}^{\prime}-N u_{02}=-N-G r \theta_{02} \\
& u_{11}^{\prime \prime}+u_{11}^{\prime}-\left(\frac{i \omega}{4}+N\right) u_{11}=-G r \theta_{11}-\frac{i \omega}{4}-N \\
& u_{12}^{\prime \prime}+u_{12}^{\prime}-\left(\frac{i \omega}{4}+N\right) u_{12}=-G r \theta_{12} \\
& \theta_{01}^{\prime \prime}+\operatorname{Pr} \theta_{01}^{\prime}=0 \\
& \theta_{02}^{\prime \prime}+\frac{4}{3}\left(\theta_{01}+C_{T}\right)^{3} \theta_{01}^{\prime \prime}+4\left(\theta_{01}+C_{T}\right)^{2} \theta_{01}^{\prime 2} \\
& +\operatorname{Pr} \theta_{02}^{\prime}=0 \\
& \theta_{11}^{\prime \prime}+\operatorname{Pr} \theta_{11}^{\prime}-\frac{i \omega}{4} \operatorname{Pr} \theta_{11}=0 \\
& \theta_{12}^{\prime \prime}+\operatorname{Pr} \theta_{12}^{\prime}-\frac{i \omega}{4} \operatorname{Pr} \theta_{12} \\
& =-\frac{4}{3}\left(\theta_{01}+C_{T}\right)^{3} \theta_{11}^{\prime}-8\left(\theta_{01}+C_{T}\right)^{2} \theta_{01}^{\prime} \theta_{11}^{\prime} \\
& -8\left(\theta_{01}+C_{T}\right)^{2} \theta_{01}^{\prime 2}-4\left(\theta_{01}+C_{T}\right)^{2} \theta_{01}^{\prime \prime} \theta_{11}
\end{aligned}
$$

with the boundary conditions

$$
\begin{aligned}
& u_{01}=0, u_{02}=0, u_{11}=0, u_{12}=0, \theta_{01}=1, \\
& \theta_{02}=0, \theta_{11}=0, \theta_{12}=0 \text { at } y=0 \\
& u_{01} \rightarrow 1, u_{02} \rightarrow 0, u_{11} \rightarrow 1, u_{12} \rightarrow 0, \\
& \theta_{01} \rightarrow 0, \theta_{02} \rightarrow 0, \theta_{11} \rightarrow 0, \theta_{12} \rightarrow 0 \text { as } y \rightarrow \infty
\end{aligned}
$$

The solutions of zeroth and first order of velocity and temperture are: 


$$
\begin{aligned}
& u_{0}(y)=1+A_{6} \mathrm{e}^{-A_{3} y}-A_{5} \mathrm{e}^{-\operatorname{Pr} y} \\
& +R\left(A_{15} \mathrm{e}^{-A_{3} y}+A_{14} \mathrm{e}^{-\operatorname{Pr} y}+A_{9} \mathrm{e}^{-4 \operatorname{Pr} y}\right. \\
& \left.+A_{10} \mathrm{e}^{-3 \operatorname{Pr} y}+A_{11} \mathrm{e}^{-2 \operatorname{Pr} y}-A_{12} y \mathrm{e}^{-\operatorname{Pr} y}\right) \\
& u_{1}(y)=1-\mathrm{e}^{-m_{2} y} \\
& +R\left(A_{11}^{\prime} \mathrm{e}^{-m_{2} y}+A_{8}^{\prime} \mathrm{e}^{-m_{1} y}+A_{9}^{\prime} \mathrm{e}^{-3 \operatorname{Pr} y}+A_{10}^{\prime} \mathrm{e}^{-2 \operatorname{Pr} y}\right) \\
& \theta_{0}(y)=\mathrm{e}^{-\operatorname{Pr} y} \\
& +R\left(A_{2} \mathrm{e}^{-\operatorname{Pr} y}-\frac{4}{9} \mathrm{e}^{-4 \operatorname{Pr} y}-2 C_{T} \mathrm{e}^{-3 \operatorname{Pr} y}\right. \\
& \left.-4 C_{T}^{2} \mathrm{e}^{-2 \operatorname{Pr} y}+\frac{4}{3} \operatorname{Pr} y C_{T}^{3} \mathrm{e}^{-\operatorname{Pr} y}\right) \\
& \theta_{1}(y)=R\left(A_{5}^{\prime} \mathrm{e}^{-m_{1} y}+A_{3}^{\prime} \mathrm{e}^{-3 \operatorname{Pr} y}+A_{4}^{\prime} \mathrm{e}^{-2 \operatorname{Pr} y}\right)
\end{aligned}
$$

The solutions for velocity and temperature distributions are:

$$
\begin{aligned}
& u(y, t)=1+A_{6} \mathrm{e}^{-A_{3} y}-A_{5} \mathrm{e}^{-\operatorname{Pr} y}+R\left(A_{15} \mathrm{e}^{-A_{3} y}+A_{14} \mathrm{e}^{-\operatorname{Pr} y}\right. \\
& \left.+A_{9} \mathrm{e}^{-4 \operatorname{Pr} y}+A_{10} \mathrm{e}^{-3 \operatorname{Pr} y}+A_{11} \mathrm{e}^{-2 \operatorname{Pr} y}-A_{12} y \mathrm{e}^{-\operatorname{Pr} y}\right) \\
& +\varepsilon \mathrm{e}^{i \omega t}\left[1-\mathrm{e}^{-m_{2} y}\right. \\
& \left.+R\left\{A_{11}^{\prime} \mathrm{e}^{-m_{2} y}+A_{8}^{\prime} \mathrm{e}^{-m_{1} y}+A_{9}^{\prime} \mathrm{e}^{-3 \operatorname{Pr} y}+A_{10}^{\prime} \mathrm{e}^{-2 \operatorname{Pr} y}\right\}\right] \\
& \theta(y, t)=\mathrm{e}^{-\operatorname{Pr} y}+R\left(A_{2} \mathrm{e}^{-\operatorname{Pr} y}-\frac{4}{9} \mathrm{e}^{-4 \operatorname{Pr} y}-2 C_{T} \mathrm{e}^{-3 \operatorname{Pr} y}\right. \\
& \left.-4 C_{T}^{2} \mathrm{e}^{-2 \operatorname{Pr} y}+\frac{4}{3} \operatorname{Pr} y C_{T}^{3} \mathrm{e}^{-\operatorname{Pr} y}\right) \\
& +\varepsilon \mathrm{e}^{i \omega t} R\left(A_{5}^{\prime} \mathrm{e}^{-m_{1} y}+A_{3}^{\prime} \mathrm{e}^{-3 \operatorname{Pr} y}+A_{4}^{\prime} \mathrm{e}^{-2 \operatorname{Pr} y}\right)
\end{aligned}
$$

\section{Skin-friction:}

The non-dimensional form of skin-friction at the plate are given by:

$$
\begin{aligned}
& C f=\tau=\left[\frac{\partial u}{\partial y}\right]_{y=0} \\
& =-A_{3} A_{6}+\operatorname{Pr} A_{5}+R \\
& {\left[-A_{3} A_{15}+\operatorname{Pr} A_{14}-4 \operatorname{Pr} A_{9}-3 \operatorname{Pr} A_{10}-2 \operatorname{Pr} A_{11}-A_{12}\right]} \\
& +\varepsilon \mathrm{e}^{i \omega t}\left[m_{2}+R\left\{-m_{2} A_{11}^{\prime}-m_{1} A_{8}^{\prime}-3 \operatorname{Pr} A_{9}^{\prime}-2 \operatorname{Pr} A_{10}^{\prime}\right\}\right]
\end{aligned}
$$

\section{Nusselt number:}

The non dimensional form of the rate of heat transfer in terms of Nusselt number at the plate are given by:

$$
\begin{aligned}
& N u=-\left[\frac{\partial \theta}{\partial y}\right]_{y=0} \\
& =\operatorname{Pr}-R\left[-A_{2} \operatorname{Pr}+\frac{16}{9} \operatorname{Pr}+6 \operatorname{Pr} C_{T}+8 C_{T}^{2} \operatorname{Pr}+\frac{4}{3} \operatorname{Pr} C_{T}^{3}\right] \\
& -\varepsilon \mathrm{e}^{i \omega t} R\left(-m_{1} A_{5}^{\prime}-3 \operatorname{Pr} A_{3}^{\prime}-2 \operatorname{Pr} A_{4}^{\prime}\right)
\end{aligned}
$$

\section{Results and Discussion}

In order to get the physical insight in to the problem, we have carried out numerical calculations for non-dimensional velocity field, temperature field, nusselt number and skin-friction have been carried out by assigning specific values to the different parameters involved in the problem, viz, Hartmann number $M$, Grashof number $G r$, Prandtl number Pr, Porosity parameter $K$, Radiation conduction parameter $\mathrm{R}$ and Temperature difference parameter $C_{T}$. The effects of these values are demonstrated through different graphs and the results are interpreated physically.

The Figures 2-4 depict the variation of velocity field $u$ against $y$ under the effect of Hartmann number $M$, Porosity parameter $K$ and radiation conduction parameter $R$. Figure 2 illustrates that the fluid velocity decreases with the increase in magnetic intensity. In other words the imposition of the transverse magnetic field tends to retard the fluid flow. This phenomenon has an excellent agreement with the physical fact that the Lorentz force generated in the present flow model due to interaction of the transverse magnetic field and the fluid velocity acts as a resistive force to the fluid flow which serves to decelerate the flow. Figures $\mathbf{3}$ and $\mathbf{4}$ demonstrate the effect of Porosity number $K$ and radiation conduction parameter $R$ on velocity field. It is simulated from the figures that an increase in Porosity parameter $K$ and radiation conduction parameter $R$ leads to a decrease in fluid velocity. i.e. the fluid motion is decelerated for increasing the permeability of the medium and for low thermal conductivity.

The Figures 5-7 exhibit the variation of temperature field $\theta$ against $y$ under the influence of temperature difference parameter $C_{T}$, radiation-conduction parameter $R$ and Prandtl number Pr. Figures 5 and 6 present the variation of Temperature difference parameter $C_{T}$ and Radiation conduction parameter $R$ on temperature field. It is

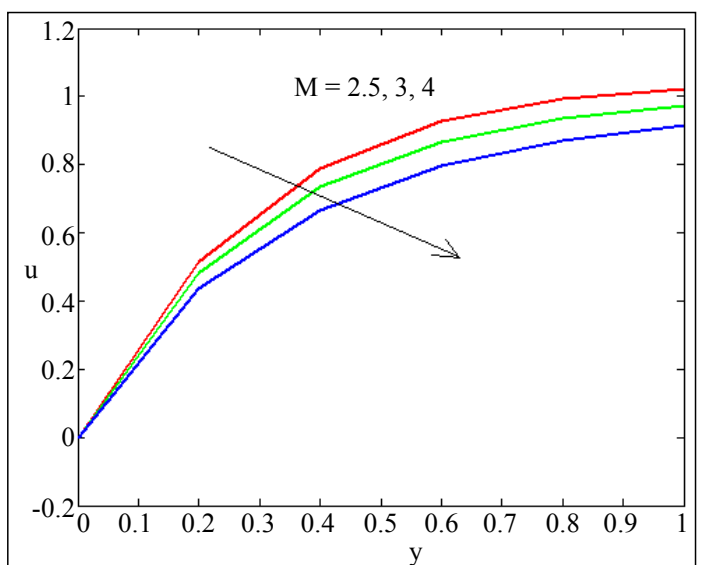

Figure 2. Velocity profile versus y for $G r=5, \operatorname{Pr}=0.71, R=$ $0.1, C t=0.01, K=1, \varepsilon=0.01, \omega t=\pi / 2$. 


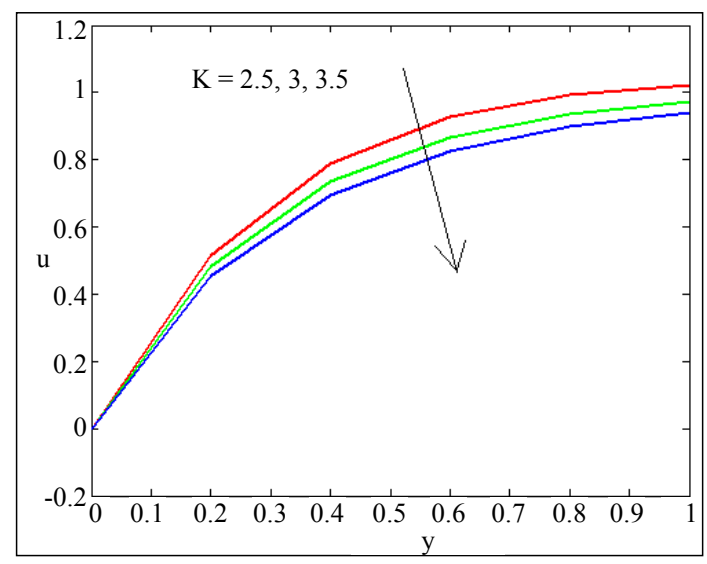

Figure 3. Velocity profile versus $y$ for $G r=5, \operatorname{Pr}=0.71, R=$ $0.1, C t=0.01, M=1, \varepsilon=0.01, \omega t=\pi / 2$.

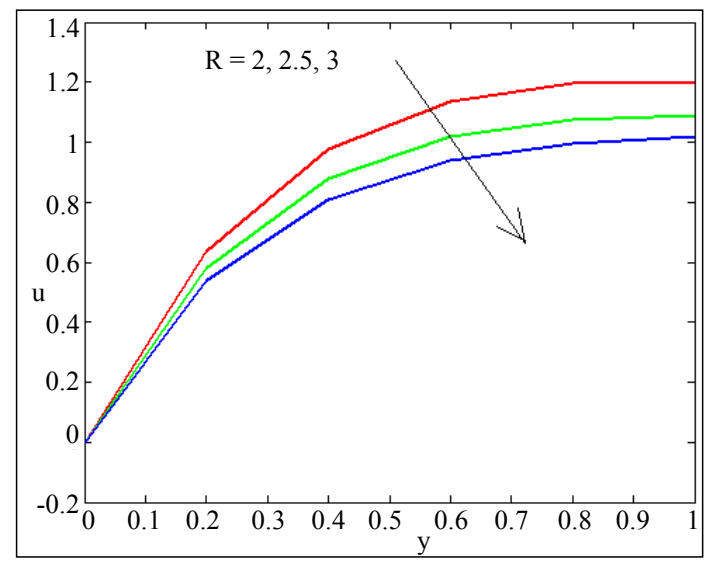

Figure 4. Velocity profile versus y for $G r=5, \operatorname{Pr}=0.71, K=$ $1, C t=0.01, M=1, \varepsilon=0.01, \omega t=\pi / 2$.

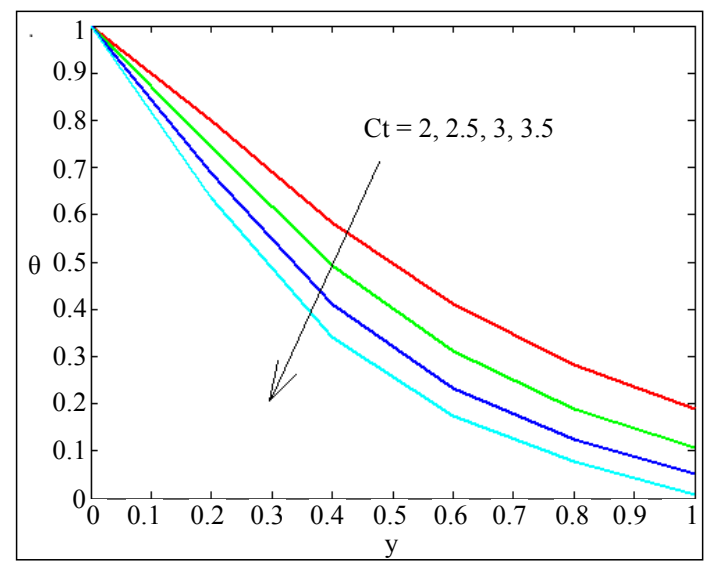

Figure 5. Temperature profile versus $y$ for $R=0.1, P r=0.71$, $\varepsilon=0.01, \omega t=\pi / 2$.

observed from both the figures that there is a steady drop in temperature due to small temperature difference and low thermal conductivity. Figure 7 indicates that the fluid temperature decreases with the increase in Prandtl number. i.e. thermal diffusivity reduces the fluid temperature.

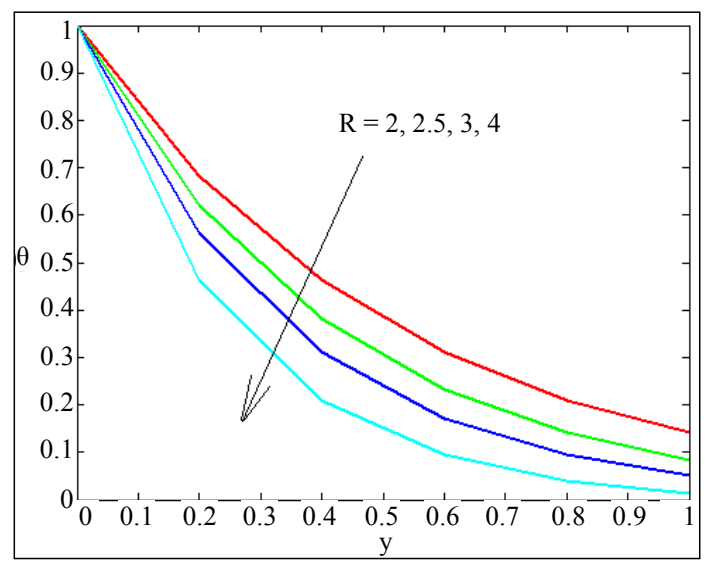

Figure 6. Temperature profile versus $y$ for $C t=0.01, \operatorname{Pr}=$ $0.71, \varepsilon=0.01, \omega t=\pi / 2$.

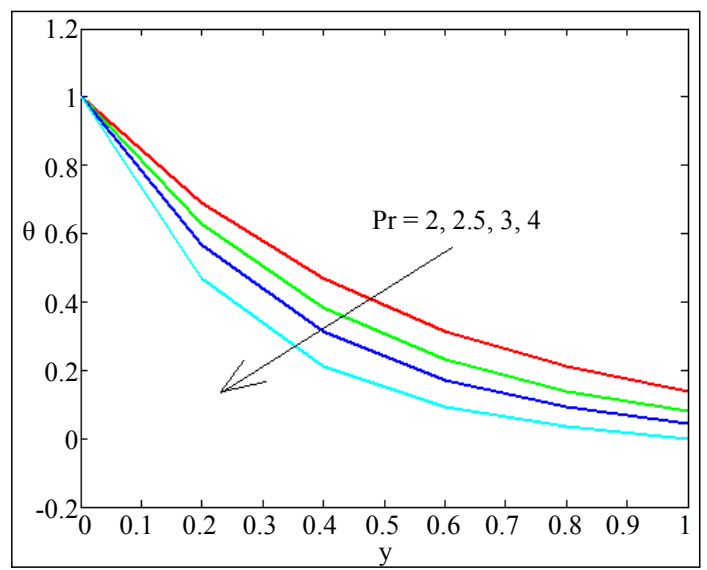

Figure 7. Temperature profile versus $y$ for $R=0.1, C t=0.01$, $\varepsilon=0.01, \omega t=\pi / 2$.

This result is in a good agreement to the fact that the thickness of the thermal boundary layer may be prevented with the increasing Prandtl number.

The effects of Prandtl number Pr, radiation-conduction parameter $R$ and temperature difference parameter $C_{T}$ on the co-efficient of rate of heat transfer in terms of Nusselt number have been displayed in Figures 8-10. It is found from the Figures 8 and 9 that the magnitude of the rate of heat transfer is considerably increased with the increase in Prandtl number Pr and Radiation-conduction parameter $R$. i.e. the energy flux is rised due to increasing the permeability of the medium as well as low thermal conductivity. Figure $\mathbf{1 0}$ predicts that the energy flux from the plate to the fluid in terms of Nusselt number get increased with the increase in Temperature difference parameter $C_{T}$.

The Figures 11-14 correspond to the co-efficient of skin-friction $\tau$ against radiation conduction parameter $R$ under the influence of magnetic parameter $M$, Porosity parameter $K$, temperature difference parameter $C_{T}$ and Grashof number $G r$. In the Figures 11, 12 and $\mathbf{1 4}$ it is 


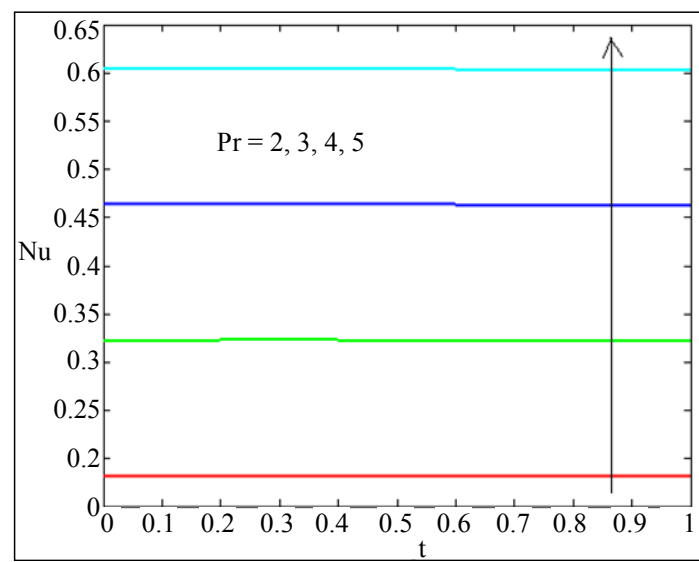

Figure 8. Nusslet number versus $t$ for $R=0.1, C t=0.01, \varepsilon=$ $0.01, \omega=1$.

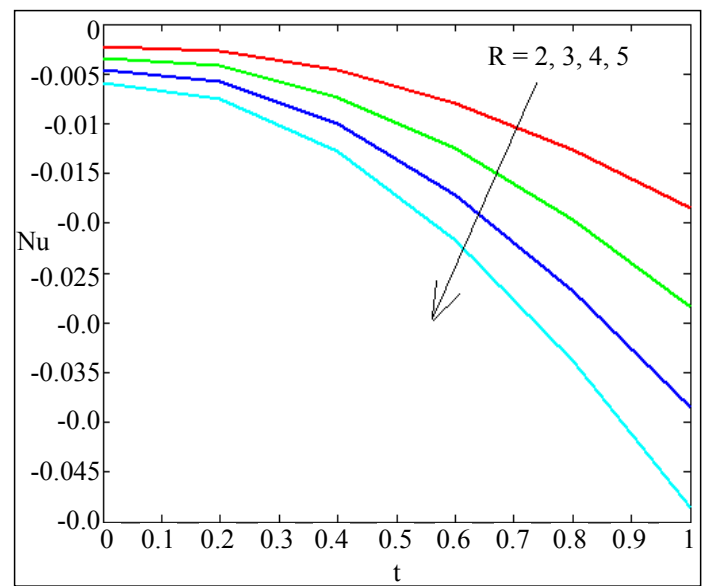

Figure 9. Nusslet number versus $t$ for $\operatorname{Pr}=0.71, C t=0.01, \varepsilon$ $=0.01, \omega=1$.

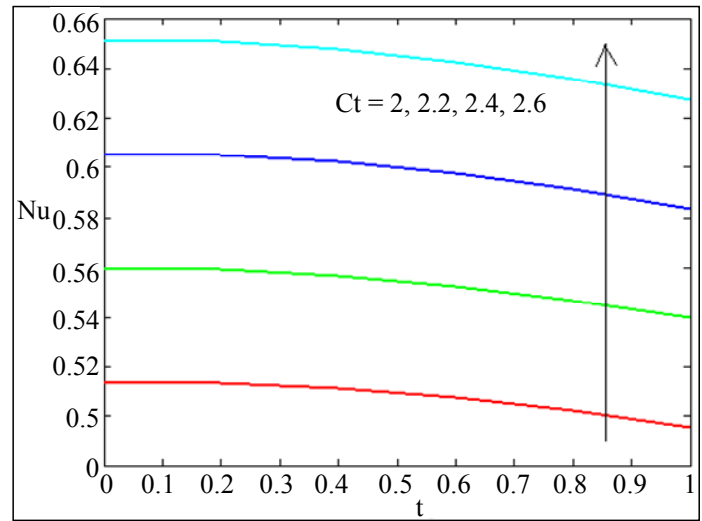

Figure 10. Nusslet number versus $t$ for $\operatorname{Pr}=0.71, R=0.1, \varepsilon$ $=0.01, \omega=1$.

observed that an increase in Magnetic parameter $M$, Porosity parameter $K$ and Grashof number $G r$ tends to raise the viscous drag whereas viscous drag falls with the increase in Temperature difference parameter $C_{T}$ in Figure 13.

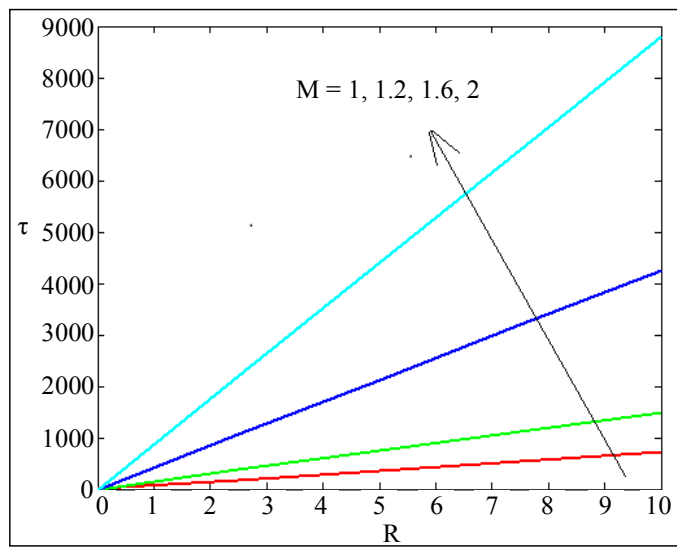

Figure 11. Co-efficient of skin friction versus $\boldsymbol{R}$ for $\boldsymbol{C t}=\mathbf{0 . 0 1}$, $\operatorname{Pr}=0.71, K=1, G r=5, \varepsilon=0.01, \omega=1, t=11 / 7$.

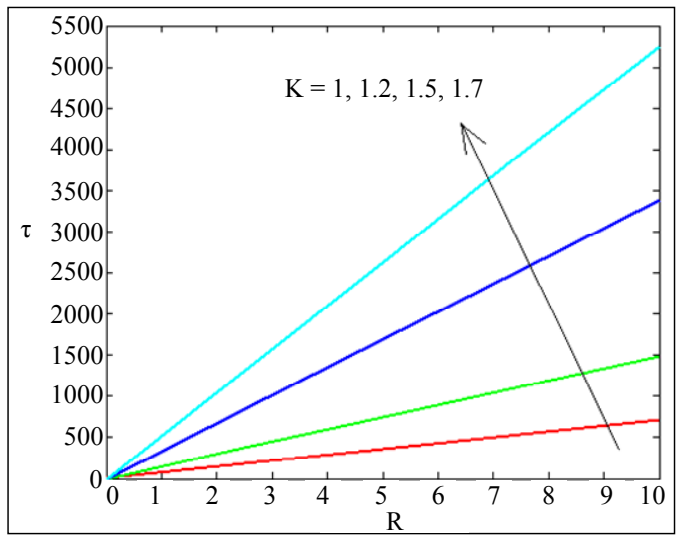

Figure 12. Co-efficient of skin friction versus $\boldsymbol{R}$ for $\boldsymbol{C t}=\mathbf{0 . 0 1}$, $\operatorname{Pr}=0.71, M=1, G r=5, \varepsilon=0.01, \omega=1, t=11 / 7$.

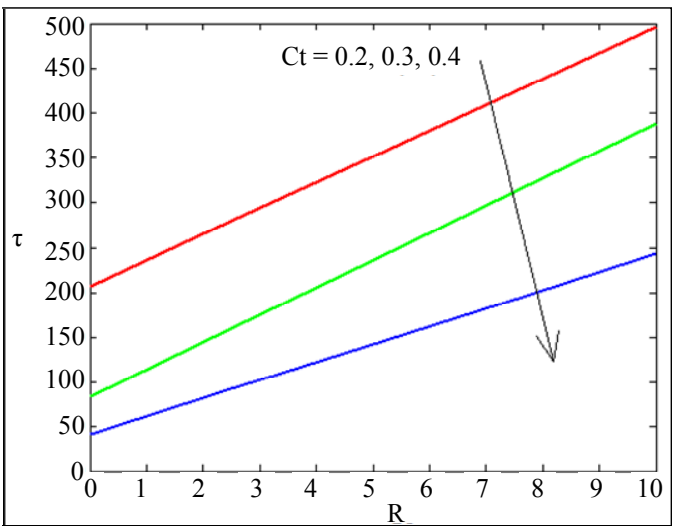

Figure 13. Co-efficient of skin friction versus $R$ for $K=1$, Pr $=0.71, M=1, G r=5, \varepsilon=0.01, \omega=1, t=11 / 7$.

\section{Conclusions}

Our investigation of the problem setup leads to the following conclusions:

- The fluid motion is decelerated under the action of tranverse magnetic field, Porosity parameter and radiation conduction parameter. 


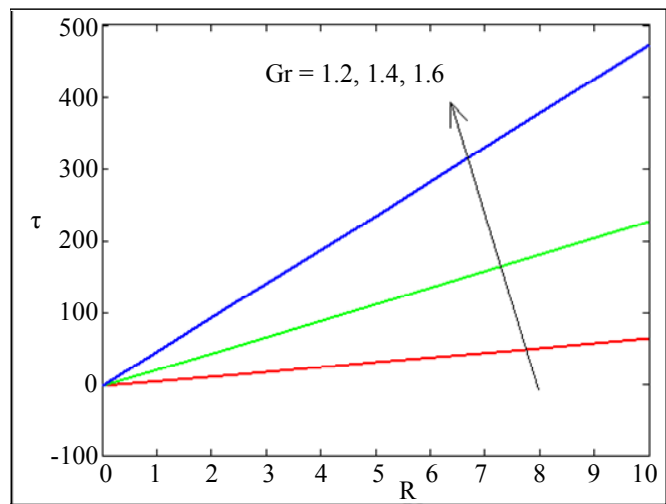

Figure 14. Co-efficient of skin friction versus $R$ for $K=0.01$, $\operatorname{Pr}=0.71, M=1, C t=5, \varepsilon=0.01, \omega=1, t=11 / 7$.

- Temperature falls due to small temperature difference and low thermal conductivity.

- The energy flux rises due to increasing the permeability of the medium as well as low thermal conductivity.

- Magnetic parameter raises the viscous drag on the plate but it falls with the increase in temperature difference parameter.

\section{Acknowledgements}

The authors are grateful to the CSIR-HRDG (India) for sponsoring this work through their Research-Grant-InAid. No. 25 (0209)/12/EMR-I.

\section{REFERENCES}

[1] H. Alfven, "Discovery of Alfven Waves," Nature, Vol. 150, No. 3805, 1942, pp. 405-406.

[2] T. G. Cowling, "Magneto Hydrodynamics," Wiley Inter Science, New York, 1957.

[3] K. P. Crammer and S. L. Pai, "Magneto-Fluid Dynamics for Engineers and Applied Physics," McGraw Hill book Co., New York, 1978.

[4] J. A. Shercliff, "A Text Book of Magneto-Hydrodynamics," Pergamon Press, London, 1965.

\section{Nomenclature}

$B_{0}=$ Strength of the applied magnetic field; $C_{T}=$ Temperature difference parameter; $G_{r}=$ Thermal Grashof number; $g=$ Acceleration due to gravit; $K_{T}=$ Thermal conductivity; $k=$ Mean absorption co-efficient; $K=$ Porosity parameter; $M=$ Local Hartmann number; $N u=$ Nusselt number; $\mathrm{Pr}=$ Prandtl number; $p^{\prime}=$ Pressure; $q_{r}$ $=$ Radiative heat flux term; $\mathrm{R}=$ Radiation-conduction parameter; $T^{\prime}=$ Dimensional temperature; $T_{w}^{\prime}=$ Dimensional temperature at the plat; $T_{\infty}^{\prime}=$ Dimensional temperature in the free stream; $U^{\prime}=$ Dimensional free
[5] V. C. A. Ferraro and C. Plumpton, "An Introduction to Magneto-Fluid Mechanics," Clarandon Press, Oxford, 1966.

[6] N. Ahmed, "MHD Free and Forced Convection with Mass Transfer from an Infinite Vertical Porous Plate," Journal of Energy, Heat and Mass Transfer, Vol. 32, No. 1, 2010, pp. 55-70.

[7] E. M. A. Elbashbeshy, "The Mixed Convection along a Vertical Plate Embedded in Non-Darcian Porous Medium with Suction and Injection," Applied Mathematics and Computation, Vol. 136, No. 1, 2003, pp. 139-149. http://dx.doi.org/10.1016/S0096-3003(02)00023-1

[8] N. P. Singh and A. K. Singh, "MHD Effects on Heat and Mass Transfer in a Flow of Viscous Fluid with Induced Magnetic Field," Indian Journal of Pure and Applied Physics, Vol. 38, No. 3, 2000, pp. 182-189.

[9] G. A. Gregantopoulos, J. Koullias, C. L. Goudas and C. Courogenis, "Free Convection and Mass Transfer Effects on the Hydromagnetic Oscillatory Flow Past an Infinite Vertical Porous Plate," Astrophysics and Space Science, Vol. 74, No. 2, 1981, pp. 357-389. http://dx.doi.org/10.1007/BF00656444

[10] M. A. Hussain and H. S. Takhar, "Radiation Effect on Mixed Convection along a Vertical Plate with Uniform Surface Temperature," Heat Mass Transfer, Vol. 31, No. 4, 1996, pp. 243-248. http://dx.doi.org/10.1007/BF02328616

[11] N. Ahmed and H. K. Sarmah, "The Radiation Effect on a Transient MHD Flow with Mass Transfer Past an Impulsively Fixed Infinite Vertical Plate," International Journal of Applied Mathematics and Mechanics, Vol. 5, No. 5, 2009, pp. 87-98.

[12] V. Rajesh and S. V. K. Varma, "Radiation Effects on MHD Flow through a Porous Medium with Variable Temperature or Variable Mass Diffusion," International Journal of Applied Mathematics and Mechanics, Vol. 6, No. 11, 2010, pp. 39-57.

[13] N. Ahmed, S. Sinha and S. Talukdar, "Dufour Effect on a Transient MHD Flow Past a Uniformly Moving Porous Plate with Heat Sink," Advances and Applications in Fluid Mechanics, Vol. 13, No. 1, 2013, pp. 1-24.

[14] M. A. Mansour, "Radiative and Free-Convection on the Oscillatory Flow Past a Vertical Plate," Astrophysics and Space Science, Vol. 166, No. 2, 1990, pp. 269-275.

stream velocity; $U_{0}=$ Mean stream velocity; $U=$ NonDimensional fluid velocity; $\left(u^{\prime}, v^{\prime}\right)=$ Components of the fluid velocity; $t^{\prime}=$ time; $\left(x^{\prime}, y^{\prime}, z^{\prime}\right)=$ Cartesian coordinates

\section{Greek Symbols}

$\rho=$ Density of the fluid; $v=$ Kinematic viscosity; $\alpha=$ Thermal diffusivity; $\omega=$ Frequency parameter; $\theta=$ Dimensionless temperature; $\sigma^{\prime}=$; tephan-Boltzmann constant; $\sigma=$ Electrical conductivity; $\beta=$ Co-efficient of volume expansion for heat transfer; $\varepsilon=$ Small reference parameter. 